\title{
CARACTERIZAÇÃO ESPECTROSCÓPICA MULTIVARIADA DO POTENCIAL ANTIOXIDANTE DE VINHOS
}

\author{
Wellington César Gallice, Iara Messerschmidt e Patricio Peralta-Zamora* \\ Departamento de Química, Universidade Federal do Paraná, CP 19081, 81531-980 Curitiba - PR, Brasil
}

Recebido em 31/3/10; aceito em 7/10/10; publicado na web em 26/1/11

\begin{abstract}
MULTIVARIATE SPECTROSCOPIC CHARACTERIZATION OF ANTIOXIDANT POTENTIAL OF WINE. In this work the antioxidant capacity of red wine samples was characterized by conventional spectroscopic and chromatographic methodologies, regarding chemical parameters like color, total polyphenolic and resveratrol content, and antioxidant activity. Additionally, multivariate calibration models were developed to predict the antioxidant activity, using partial least square regression and the spectral data registered between 400 and $800 \mathrm{~nm}$. Even when a close correlation between the evaluated parameters has been expected many inconsistencies were observed, probably on account of the low selectivity of the conventional methodologies. Models developed from mean-centered spectra and using 4 latent variables allowed high prevision capacity of the antioxidant activity, permitting relative errors lower than $3 \%$.
\end{abstract}

Keywords: wine; antioxidant potential; multivariate electronic spectroscopy.

\section{INTRODUÇÃO}

Estudos relativamente recentes demonstram que, mesmo adepto a uma dieta rica em gorduras, o povo francês apresenta uma baixa incidência de doenças coronárias, assim como uma baixa tendência à obesidade. ${ }^{1}$ Este fato, universalmente conhecido como o paradoxo francês, é atribuído ao consumo diário de vinho, produto que, em função da sua rica composição em espécies polifenólicas, exerce um importante efeito antioxidante. ${ }^{2}$

Grande parte das reações metabólicas do organismo humano é realizada em meio aeróbico (ex. respiração mitocondrial), o que irremediavelmente leva à geração de inúmeras espécies reativas de oxigênio (EROs), com destaque para radicais peroxila (ROO*), alcoxila $\left(\mathrm{RO}^{\bullet}\right)$, hidroxila $\left(\mathrm{HO}^{\bullet}\right)$, superóxido $\left(\mathrm{O}_{2}^{*}\right)$ e oxigênio singlete $\left({ }^{1} \mathrm{O}_{2}\right)$, dentre outras. Normalmente, o organismo controla a concentração deste tipo de espécies, principalmente por meio da produção de enzimas que catalisam reações de inativação de radicais. ${ }^{3}$ Contudo, o aumento da geração intracelular de radicais ou a deficiência nos mecanismos antioxidantes podem desequilibrar este sistema de controle, ${ }^{4}$ produzindo o denominado estresse oxidativo. Em geral, estima-se que o estresse oxidativo esteja envolvido na incidência de inúmeras doenças, dentre as quais se podem destacar câncer, arteriosclerose, reumatismo, artrite, artrose e doenças degenerativas, como Parkinson e Alzheimer, dentre outras.

Em função da comprovada relação existente entre o estresse oxidativo e o surgimento de patologias características, grande ênfase tem sido dada nos últimos anos ao consumo de alimentos que apresentem atividade antioxidante. Dentro deste contexto, destaque deve ser dado ao vinho, principalmente tinto, produto que apresenta elevada ação antioxidante, em razão da presença de altas concentrações de substâncias polifenólicas, como taninos, ácidos fenólicos, flavonoides, catequinas e antocianidinas. ${ }^{5}$ Mesmo que a composição polifenólica dos vinhos seja altamente dependente do tipo de uva, das condições climáticas, das técnicas de cultivo e produção e da forma de armazenamento, estima-se que todos os tipos de vinho exerçam uma importante ação protetora contra doenças cadiovasculares e degenerativas. ${ }^{6}$

*e-mail: zamora@quimica.ufpr.br
Dentro do extenso grupo de espécies fenólicas responsáveis pela ação antioxidante do vinho, tem sido dado destaque ao resveratrol (trans-3,5,4'-tri-hidroxistilbeno), um estilbeno que apresenta vários efeitos biológicos de relevância, com destaque para a atividade anticâncer e cardioprotetora. ${ }^{7}$

A avaliação do potencial antioxidante de vinhos pode ser realizada pela quantificação de uma substância específica (ex. resveratrol), pela quantificação de uma família de substâncias (ex. polifenóis totais) ou pela avaliação da capacidade antioxidante frente a uma espécie radicalar padrão. De maneira geral, o teor de resveratrol é determinado por cromatografia em fase líquida, usualmente utilizando-se injeção direta. ${ }^{8}$ Por sua vez, o teor de espécie polifenólicas totais costuma ser determinado espectrofotometricamente, utilizando-se, por exemplo, o reagente de Folin-Ciocalteau. ${ }^{9}$ A capacidade antioxidante pode ser determinada por vários métodos disponíveis. Entretanto, as técnicas mais frequentemente empregadas envolvem a inativação de espécies radicalares, usualmente o radical cátion $\mathrm{ABTS}^{+}$, resultante da reação entre ABTS (2,2'-azino-bis(3-etilbenzotiazolina-6-ácido sulfônico) e persulfato de potássio. ${ }^{10}$

Embora uma importante parcela da atividade antioxidante de vinhos seja atribuída à presença de espécies polifenólicas, grande parte das quais apresenta absorção característica na região espectral compreendida entre 400 e $800 \mathrm{~nm}$, não existem relatos na literatura sobre a avaliação deste parâmetro recorrendo-se à espectroscopia eletrônica. Sendo assim, o presente trabalho apresentou como principal objetivo avaliar a capacidade antioxidante de vinhos, utilizando dados espectrais e procedimentos de calibração multivariada fundamentados em regressão por mínimos quadrados parciais (PLS).

\section{PARTE EXPERIMENTAL}

\section{Amostras de vinho}

Grande parte das 47 amostras de vinho tinto (Tabela 1) foi adquirida em supermercados, distribuidoras de bebidas e adegas da região (Curitiba-PR). Outras foram gentilmente doadas pelas vinícolas Miolo (RS), Garibaldi (RS) e Campo Largo (PR). 
Tabela 1. Descrição das principais características dos vinhos tintos utilizados no estudo

\begin{tabular}{|c|c|c|c|c|}
\hline Amostra & Vinícola & Uva & Ano & Nacionalidade \\
\hline A01 & Do Avô & Mistura & 2006 & Brasil \\
\hline $\mathrm{A} 02$ & Carta Nueva & Cabernet Sauvignon & 2008 & Chile \\
\hline $\mathrm{A} 03$ & Diego del Valle & Carmenere & 2000 & Chile \\
\hline A04 & Franco Italiano & Mistura - Colonial & 2008 & Colombo-Brasil \\
\hline A05 & Salton Classic & Merlot & 2007 & Brasil \\
\hline A06 & Aurora Varietal & Pinot Noir & 2008 & Brasil \\
\hline A07 & Chalet Du Clermont & Mistura & 2005 & Brasil \\
\hline A08 & Acquasantiera & Cabernet Sauvignon & 2005 & Brasil \\
\hline A09 & $\begin{array}{c}\text { Santa Carolina - } \\
\text { Reservado }\end{array}$ & Cabernet Sauvignon & 2007 & Chile \\
\hline A10 & $\begin{array}{c}\text { Miolo } \\
\text { (Campos de Cima) }\end{array}$ & Merlot & 2008 & Brasil \\
\hline A11 & $\begin{array}{c}\text { Miolo } \\
\text { (Campanha Gaucha) }\end{array}$ & Merlot & 2008 & Brasil \\
\hline A12 & Santa Florentina & Malbec & 2007 & Argentina \\
\hline A13 & Santa Helena & Cabernet Sauvignon & 2007 & Chile \\
\hline A14 & Santa Florentina & Cabernet Sauvignon & 2007 & Argentina \\
\hline A 15 & $\begin{array}{c}\text { Miolo } \\
\text { (Vale dos Vinhedos) }\end{array}$ & Merlot & 2008 & Brasil \\
\hline A16 & Saint Germain & Merlot & 2008 & Brasil \\
\hline A17 & Vinãs de Alvear & Malbec & 2008 & Argentina \\
\hline A18 & Da Casa - Garibaldi & Isabel e Bordo & 2008 & Brasil \\
\hline A19 & Marcus James & Tannat & 2008 & Brasil \\
\hline A20 & Trapiche & Malbec & 2007 & Argentina \\
\hline A21 & Etchart & Malbec & 2006 & Argentina \\
\hline A22 & Meridien 68 & Cabernet Sauvignon & 2008 & Argentina \\
\hline A23 & Caballo Negro & Carmenere & 2007 & Chile \\
\hline A24 & Trimento Tribu & Pinot Noir & 2007 & Chile \\
\hline A25 & Santa Helena & Malbec & 2007 & Argentina \\
\hline A26 & Trivento Tribu & Tempranillo & 2007 & Argentina \\
\hline A27 & Evoluccion & Merlot & 2006 & Chile \\
\hline A28 & Solar del Paso & Tannat & 2007 & Uruguai \\
\hline A29 & Caballo de Oro & Syrah & 2005 & Chile \\
\hline A30 & Vapore 1888 & Cabernet Sauvignon & 2005 & Brasil \\
\hline A31 & Baccio & Cabernet Sauvignon & 2006 & Brasil \\
\hline A32 & Trapiche & Merlot & 2007 & Argentina \\
\hline A33 & Leonardo Falcone & Tannat & 2004 & Uruguai \\
\hline A34 & Carta Vieja & Cabernet Sauvignon & 2007 & Chile \\
\hline A35 & Norton & Bonarda & 2006 & Argentina \\
\hline A36 & Terrasul & Cabernet Sauvignon & 2004 & Brasil \\
\hline A37 & Norton & Barbera & 2007 & Argentina \\
\hline A38 & Colina del Vale & Mistura & 2008 & Brasil \\
\hline A39 & Terranova & Shiraz & 2006 & Brasil \\
\hline A40 & Trapiche & Malbec & 2008 & Argentina \\
\hline A41 & Espiritu de Chile & Carmenere & 2007 & Chile \\
\hline A42 & Castilo de Liria & Bobal\&Shiraz & 2005 & Espanha \\
\hline A43 & Latitud 33 & Syrah & 2007 & Argentina \\
\hline
\end{tabular}

Tabela 1. Continuação

\begin{tabular}{ccccc}
\hline Amostra & Vinícola & Uva & Ano & Nacionalidade \\
A44 & Santa Helena & Malbec & 2007 & Argentina \\
A45 & Veneto & Merlot & 2006 & Itália \\
A46 & Calvet & Merlot & 2005 & França \\
A47 & Obikwa & Shiraz & 2006 & África do Sul \\
\hline
\end{tabular}

\section{Caracterização espectroscópica}

Espectros na região do visível $(400-800 \mathrm{~nm})$ foram registrados num espectrofotômetro Shimadzu modelo UV-2401PC, empregando cela de quartzo com caminho ótico de $1,0 \mathrm{~cm}$. As amostras de vinho foram diluídas em água destilada (diluição de 10 vezes), filtradas em membrana de acetato de celulose $(0,45 \mu \mathrm{m})$ e medidas diretamente.

A partir dos dados espectrais foram calculados o índice de cor (IC, Equação 1) e a tonalidade (T, Equação 2), de acordo com metodologia descrita por Glories e citada por Castillo-Sánchez. ${ }^{11}$

$$
\begin{gathered}
\mathrm{IC}=\text { Ab. } 420 \mathrm{~nm}+\text { Ab. } 520 \mathrm{~nm}+\text { Ab. } 620 \mathrm{~nm} \\
\mathrm{~T}=\text { Ab. } 420 / \text { Ab. } 520
\end{gathered}
$$

\section{Determinação cromatográfica de resveratrol}

Resveratrol foi determinado por cromatografia em fase líquida de alta eficiência, de acordo com procedimento descrito por Souto e colaboradores, ${ }^{8}$ utilizando-se cromatógrafo Waters equipado com coluna C18 (4,6 x $150 \mathrm{~mm}$, partícula de $5 \mu \mathrm{m})$ e detector por arranjo de fotodiodos. A eluição foi realizada no modo gradiente, utilizandose metanol:ácido acético:água na proporção 10:2:8 (Fase A) e 90:2:8 (Fase B). A eluição seguiu a programação 100 a $85 \%$ de Fase A nos 15 primeiros min, 85 a $50 \%$ nos próximos 10 min e 50 até $30 \%$ nos próximos 9 min e 30 a $0 \%$ nos 11 min finais.

Amostras de 100,00 mL de vinho foram extraídas com 4 porções de $10,0 \mathrm{~mL}$ de acetato de etila. As fases orgânicas foram reunidas, levadas até secura e dissolvidas em 10,00 mL de solução aquosa de etanol $(10 \% \mathrm{v} / \mathrm{v})$. Após filtração em membrana de nitrato de celulose (Millipore ${ }^{\circledR}, 0,45 \mu \mathrm{m}$ ), as amostras foram analisadas por cromatografia.

A determinação de resveratrol foi viabilizada por padronização externa, utilizando-se uma curva analítica elaborada com padrão de resveratrol (Sigma), na faixa de concentração compreendida entre 0,5 e $7,0 \mathrm{mg} \mathrm{L}^{-1}(\mathrm{n}=8)$.

\section{Determinação de fenóis totais}

A determinação de formas fenólicas totais foi realizada pelo método de Folin-Ciocalteau, ${ }^{2,5,9}$ que se fundamenta na redução dos ácidos fosfomolibídico e fosfotunguístico na presença de formas fenólicas, com formação de complexos de coloração azul que absorvem fortemente entre 620 e $700 \mathrm{~nm}$.

Porções de $1,00 \mathrm{~mL}$ da amostra de vinho foram transferidas para um balão volumétrico de $100,00 \mathrm{~mL}$, completando-se o volume com água destilada. Uma alíquota de $1,00 \mathrm{~mL}$ desta solução foi adicionada de $500 \mu \mathrm{L}$ do reagente de Folin-Ciocalteau $\left(2,0 \mathrm{~mol} \mathrm{~L}^{-1}\right)$ e $2,5 \mathrm{~mL}$ de água destilada e agitada vigorosamente. Após $10 \mathrm{~min}$ foi adicionado 1,0 $\mathrm{mL}$ de $\mathrm{Na}_{2} \mathrm{CO}_{3}(20 \% \mathrm{~m} / \mathrm{v})$, agitando-se por $30 \mathrm{~s}$. A mistura foi mantida em local escuro durante $2 \mathrm{~h}$, registrando-se a absorvância em $760 \mathrm{~nm}$.

Curvas analíticas foram elaboradas entre 0,25 e $2,5 \mathrm{~g} \mathrm{~L}^{-1}$, utilizando-se solução padrão de ácido gálico. Devido à heterogeneidade estrutural dos compostos fenólicos, os resultados foram expressos em equivalentes de ácido gálico. 


\section{Determinação da atividade antioxidante}

A determinação da atividade antioxidante foi realizada por espectroscopia na região do visível, utilizando-se método fundamentado na inativação do radical ABTS.+ ${ }^{+}$Equação 3$) .{ }^{12} \mathrm{O}$ referido radical foi formado através da reação entre $5,0 \mathrm{~mL}$ da solução estoque de ABTS $\left(7,0 \times 10^{-3} \mathrm{~mol} \mathrm{~L}^{-1}\right.$, Acros $)$ e $88 \mu \mathrm{L}$ de solução de persulfato de potássio $\left(0,14 \mathrm{~mol} \mathrm{~L}^{-1}\right)$. A mistura foi mantida no escuro durante $18 \mathrm{~h}$, sendo posteriormente diluída em álcool etílico até absorvância de 0,80 \pm 0,05 em $734 \mathrm{~nm}$ (diluição típica de 1,0 mL em $100 \mathrm{~mL}$ ).

Para traçar a curva analítica utilizou-se solução etanólica de Trolox (6-Hidroxi-2,5,7,8-tetrametilcroman-2-ácido carboxílico, Sigma), um antioxidante análogo à vitamina $\mathrm{E}$, na faixa de concentração compreendida entre $1,0 \times 10^{-4} \mathrm{e} 2,0 \times 10^{-3} \mathrm{~mol} \mathrm{~L}^{-1}(\mathrm{n}=5)$. Em ambiente escuro, $30 \mu \mathrm{L}$ da solução de Trolox foram adicionadas a $3,0 \mathrm{~mL}$ da solução de radical $\mathrm{ABTS}^{+}$, agitando-se durante $20 \mathrm{~s}$. Após $6 \mathrm{~min}$, a leitura foi realizada em $734 \mathrm{~nm}$.

$\mathrm{Na}$ análise das amostras de vinho foi utilizado um procedimento idêntico, utilizando-se amostras diluídas 5 vezes $(2,00 \rightarrow 10,00 \mathrm{~mL})$.

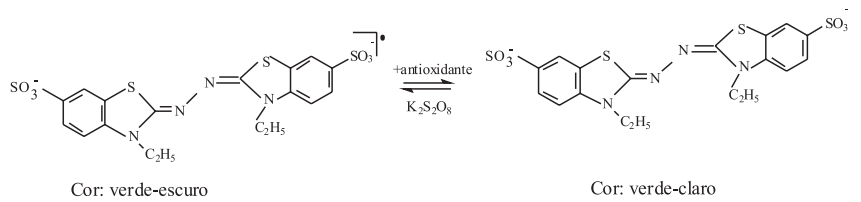

\section{Estudos quimiométricos}

Estudos quimiométricos foram realizados por rotinas de análise de componentes principais (PCA) e regressão por mínimos quadrados parciais (PLS), ambas executadas em ambiente Matlab (versão 6.5) com funções PLS-toolbox.

Para construção dos modelos de calibração multivariada foram utilizados os dados espectrais obtidos na região do visível (400$800 \mathrm{~nm}$ ), os quais foram correlacionados com a concentração de resveratrol, polifenóis totais e atividade antioxidante, parâmetros estes obtidos pelos métodos de referência. Diversos sistemas de préprocessamento de sinais foram avaliados, dentre os quais se destacam dados centrados na média, alisados e derivados.

O conjunto total de espectros (47 amostras) foi dividido em dois subconjuntos: um de calibração $(n=42)$ e outro de validação externa ( $\mathrm{n}=5)$, este último composto pelas amostras A3, A16, A29, A37 e A44. O número de variáveis latentes foi selecionado a partir da análise dos valores de erro de previsão na etapa de validação cruzada (RMSEV: root mean square error of cross validation, Equação 4), realizada por uma rotina de leave-one-out. A capacidade de previsão dos modelos foi avaliada em função dos erros de previsão (RMSEP) associados ao conjunto de validação externa.

$$
\operatorname{RMSEV}(\text { ou RMSEP })=\left[\Sigma\left(\mathrm{y}_{\text {previsto }}-\mathrm{y}_{\text {referência }}\right)^{2} / \mathrm{n}\right]^{1 / 2}
$$

onde: $n$ é o número de amostras, $y_{\text {referência }}$ são os valores determinados pelo método de referência e $\mathrm{y}_{\text {previsto }}$ são os valores previstos pelo modelo PLS.

\section{RESULTADOS E DISCUSSÃO}

\section{Intensidade de cor (IC) e tonalidade (T)}

O vinho corresponde a uma complexa mistura de substâncias, o que faz com que seja bastante difícil estabelecer correlações entre a sua cor e composição química. ${ }^{13}$ Mesmo assim, estima-se que a intensidade e a tonalidade da cor estejam estreitamente relacionadas à presença de pigmentos hidrossolúveis de natureza fenólica, principalmente antocianinas, e à formação de produtos de condensação durante o seu envelhecimento. ${ }^{14}$ Desta forma, a coloração do vinho tinto pode mudar de vermelho-azulada (vinhos jovens) à marromavermelhada (vinhos maduros), basicamente em função da formação de pigmentos poliméricos, por reação entre antocianinas e outras formas fenólicas. ${ }^{15}$

De maneira geral, estima-se que as reações que caracterizam o envelhecimento do vinho podem ser evidenciadas por modificações no espectro de absorção na região do visível, particularmente nos comprimentos de onda considerados no cálculo do índice de cor (420, 520 e $620 \mathrm{~nm}$ ). Nos espectros apresentados na Figura 1 é possível verificar perfis de absorção similares para grande parte das amostras. Outras, entretanto, apresentam elevada absorção na região monitorada (10, 11 e 39), ao mesmo tempo em que apresentam elevados valores de IC (Figura 2). Um comportamento diferenciado também pode ser observado nas amostras 6, 16, 17 e 18, as quais apresentam baixa intensidade de absorção e, consequentemente, baixos valores de IC.

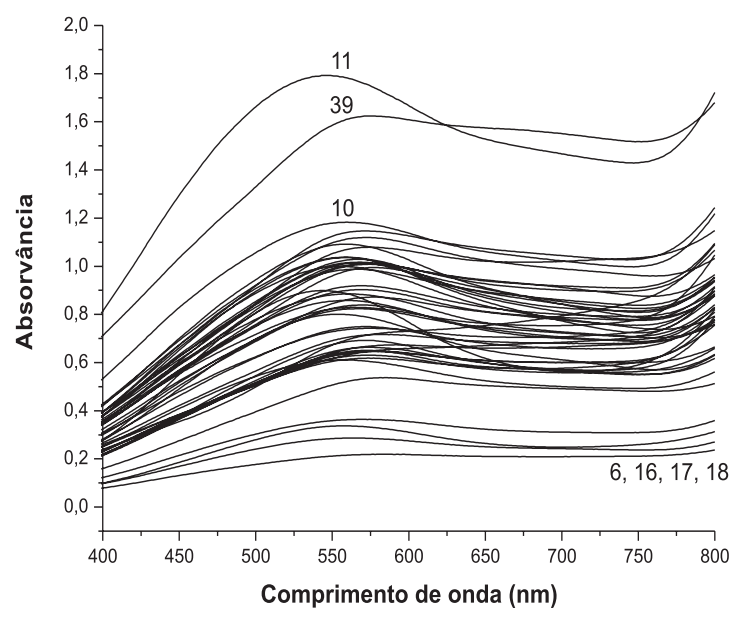

Figura 1. Espectros eletrônicos (400-800 nm) das amostras de vinho utilizadas no estudo

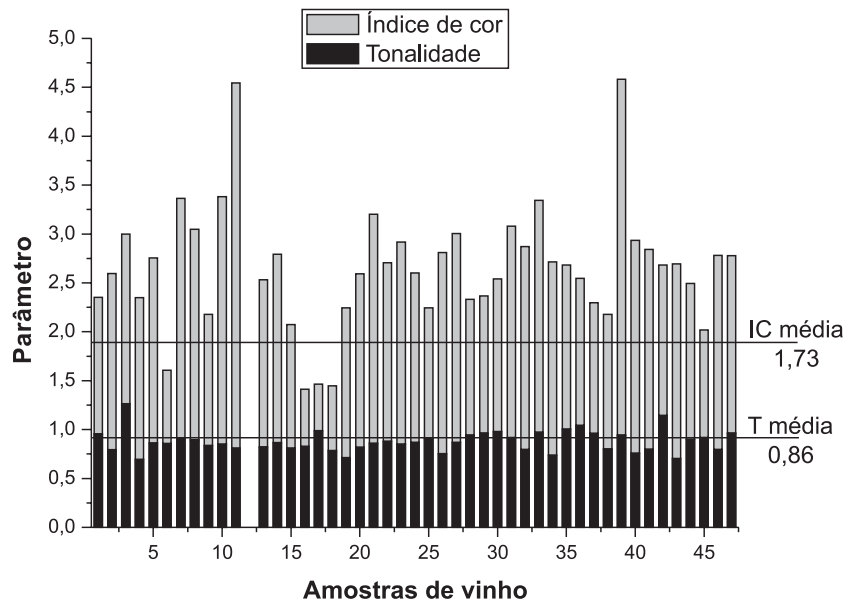

Figura 2. Representação gráfica dos valores índice de cor (IC: Ab. $420 \mathrm{~nm}$ + Ab. $520 \mathrm{~nm}+$ Ab. $620 \mathrm{~nm})$ e de tonalidade (T: Ab. $420 \mathrm{~nm} / \mathrm{Ab} .520 \mathrm{~nm}) \mathrm{nas}$ amostras de vinho analisadas

Em teoria, os valores de IC deveriam servir de subsídio para a caracterização ou classificação de amostras de vinho, principalmente em relação ao grau de envelhecimento. Entretanto, esta avaliação não pode ser convenientemente realizada levando-se em consideração 
apenas os valores de absorção em comprimentos de onda característicos, em função da interferência provocada pelas inúmeras espécies químicas presentes no vinho. As amostras 10 e 11, por exemplo, mesmo apresentando os maiores valores de IC, pertencem à mesma safra que as amostras 6,16,17 e 18, que apresentam os mais baixos valores deste parâmetro.

Objetivando verificar a existência de semelhanças e diferenças espectrais que pudessem ser correlacionadas com a safra ou com o grau de maturidade dos vinhos, os dados espectrais foram processados por uma rotina de PCA. O processamento dos dados espectrais (400$800 \mathrm{~nm}$ ) centrados na média permitiu verificar que duas componentes principais (CPs) são capazes de representar aproximadamente $94 \%$ da variância dos dados espectrais. No gráfico de escores (Figura 3) é possível observar certa distinção entre as amostras de vinhos mais "velhos" (safras de 2000, 2004, 2005 e 2006) e mais "novos" (safras de 2007 e 2008), diferenciação esta que é viabilizada pela segunda componente principal. Uma vez que o gráfico de pesos (loadings) para a segunda CP (Figura 1S, material suplementar) mostra grande relevância da região espectral centrada em $550 \mathrm{~nm}$, é possível assumir que a diferenciação observada é uma função da presença de produtos de condensação entre antocianinas e catequinas. ${ }^{16}$

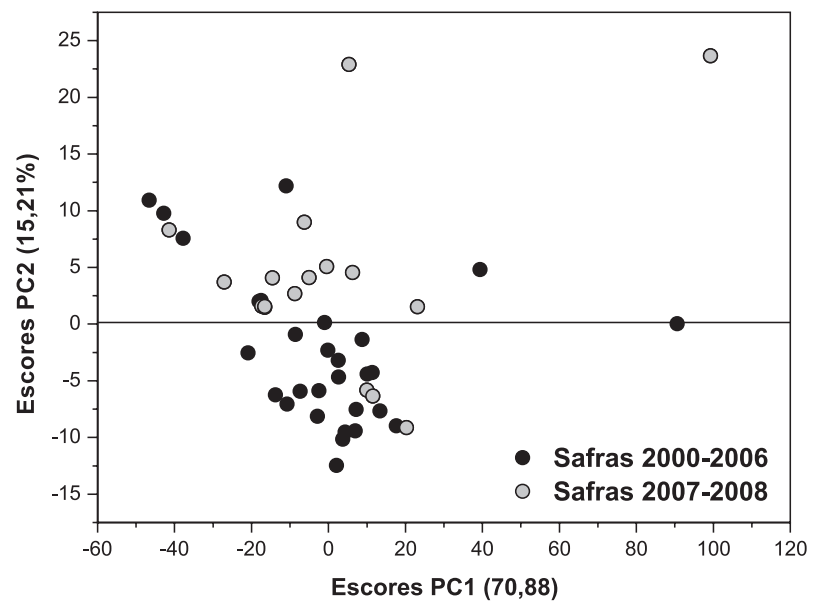

Figura 3. Gráfico de escores para as duas primeiras componentes principais (PC1 e PC2) na análise de dados espectrais por rotina de PCA

Utilizando-se outras componentes principais é possível observar alguns agrupamentos envolvendo vinhos do mesmo varietal (Figura 2S, material suplementar). Neste caso, o critério não corresponde apenas aos comprimentos de onda usualmente utilizados para avaliar índice de cor e tonalidade (420, 520 e $620 \mathrm{~nm})$, mas praticamente toda a região espectral monitorada (Figura $1 \mathrm{~S}$, material suplementar). Novamente, a existência de amostras de um mesmo varietal classificadas em regiões diferentes indica que a informação espectral monitorada não reúne todos os argumentos necessários para uma adequada diferenciação dos vinhos, levando-se em consideração o grau de envelhecimento.

\section{Concentração de polifenóis totais}

Inicialmente, a repetibilidade da metodologia foi avaliada por análise em quintuplicata de uma mesma amostra de vinho e por análise em triplicata de 7 amostras diferentes. Em todos os casos foi observado um desvio padrão relativo médio inferior a $3 \%$, o que demonstra uma boa precisão da metodologia utilizada.

A concentração de polifenóis totais nas 47 amostras analisadas é apresentada na Figura 4. Os valores limites foram 0,97 (amostra 45) e 2,91 (amostra 11), com valor médio de 1,91 $\mathrm{g} \mathrm{L}^{-1}$. Embora a concentração de compostos fenólicos varie consideravelmente, em função da variedade da uva, dos fatores ambientais no vinhedo, das técnicas de processamento e das condições de armazenamento (maturação), ${ }^{9}$ os referidos resultados mostram-se coerentes com os resultados relatados na literatura para vinhos similares., ${ }^{2,17}$

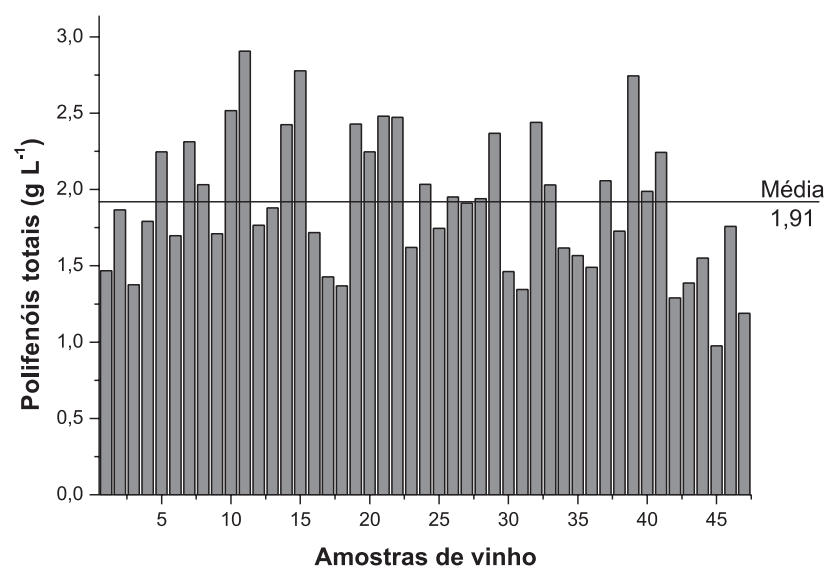

Figura 4. Representação gráfica da concentração de polifenóis totais nas amostras de vinho analisadas

As amostras 11 e 39 apresentam as maiores concentrações de fenóis totais (Figura 4), ao mesmo tempo em que mostram elevada intensidade de absorção (Figura 1) e elevados valores de índice de cor (Figura 2). Por sua vez, as amostras 16, 17 e 18 apresentam as menores concentrações de fenóis totais, assim como baixos valores de absorção e intensidade de cor. Estas observações sugerem, pelo menos em primeira análise, que os dados espectrais podem fornecer informação relevante para a elaboração de modelos que objetivem a quantificação de formas fenólicas.

Neste trabalho, modelos multivariados de calibração foram elaborados a partir de dados espectroscópicos (400-800 nm), utilizando-se regressão por mínimos quadrados parciais e diversos sistemas de préprocessamento de sinais. Os modelos foram desenvolvidos a partir de um conjunto de calibração composto por 42 amostras, sendo que as 5 amostras restantes (A3, A16, A29, A37 e A44) foram reservadas para validação externa do modelo.

Utilizando-se 4 variáveis latentes, que representam $44,21 \%$ da variância da matriz de concentração e 99,83\% da variância da matriz de espectros, os modelos permitiram a obtenção de erros de previsão (validação externa) da ordem de $15 \%$, com pouca influência dos sistemas de pré-processamento utilizados (Tabela 2). A presença de amostras anômalas foi verificada pela avaliação dos parâmetros resíduos de Student e Leverage (resultados não apresentados). Nesta avaliação, a amostra 15 apresentou um valor de resíduo superior ao limite (2,5), enquanto que as amostras 4 e 11 apresentaram elevados valores de Leverage (maior que 0,29). Em função deste fato, novos modelos foram desenvolvidos na ausência destas amostras, com resultados praticamente idênticos aos relatados anteriormente.

Explicar a falta de ajuste representa uma tarefa difícil, principalmente levando-se em consideração a extrema complexidade química das amostras de vinho. De qualquer forma, estima-se que grande parte da discrepância seja devida à pouca seletividade da metodologia de Folin-Ciocalteau, a qual está sujeita à interferência por parte de espécies redutoras de caráter não fenólico. Este tipo de limitação foi muito bem destacada em recente publicação, ${ }^{18}$ na qual se salienta a baixa seletividade da técnica e a significativa interferência por parte de substâncias naturalmente presentes em produtos vegetais, como vitamina $\mathrm{C}$, bissulfito e xantinas. 
Tabela 2. Previsão do teor de fenóis totais do conjunto de validação externa utilizando-se modelos desenvolvidos com 4 variáveis latentes e espectros centrados na média $(\mathrm{CM})$, em primeira derivada ( $1^{\mathrm{a}}$ deriv. $)$ e em segunda derivada $\left(2^{\mathrm{a}}\right.$ deriv. $)$

\begin{tabular}{cccccccc}
\hline Amostra & $\begin{array}{c}\text { Fenóis totais } \\
\left(\mathrm{g} \mathrm{L}^{-1}\right)\end{array}$ & $\mathrm{CM}$ & Erro (\%) & $1^{\text {a }}$ deriv. & Erro (\%) & $2^{\text {a deriv. }}$ & Erro (\%) \\
\hline A3 & 1,38 & 1,18 & $-14,49$ & 1,31 & $-5,07$ & 1,41 & 2,17 \\
A16 & 1,72 & 1,43 & $-16,86$ & 1,44 & $-16,27$ & 1,34 & $-22,09$ \\
A29 & 2,37 & 1,60 & $-32,48$ & 1,63 & $-31,22$ & 1,61 & $-32,06$ \\
A37 & 2,06 & 1,78 & $-13,59$ & 1,65 & $-19,90$ & 1,71 & $-16,99$ \\
A44 & 1,55 & 1,65 & 6,45 & 1,63 & 5,16 & 1,61 & 3,87 \\
Erro Médio(\%)* & & & 16,77 & & & 15,52 & \\
\hline
\end{tabular}

${ }^{*} \operatorname{Erro}(E)=\frac{x_{i}-x}{x} * 100, \bar{E}=\frac{\sum|E|}{n}$

\section{Concentração de resveratrol}

Inicialmente, a precisão (repetibilidade) da metodologia foi avaliada por análise em triplicata de 3 amostras de vinho. Os valores médios encontrados nesta avaliação foram de 0,68 a $1,33 \mathrm{mg} \mathrm{L}^{-1}$, com desvios médios da ordem de $6 \%$. Desvios desta magnitude são superiores aos apresentados por metodologias cromatográficas fundamentadas em injeção direta das amostras. ${ }^{19}$ Entretanto, mostram-se inferiores aos valores apresentados por metodologias precedidas por procedimentos de extração em fase sólida. ${ }^{20}$

Os resultados encontrados na análise das 47 amostras de trabalho são apresentados na Figura 5. Embora o intervalo de concentração encontrada esteja entre 0,14 e $5,75 \mathrm{mg} \mathrm{L}^{-1}$, com valor médio da ordem de $1,8 \mathrm{mg} \mathrm{L}^{-1}$, grande parte das amostras apresentam concentração entre aproximadamente 1,0 e 2,0 $\mathrm{mg} \mathrm{L}^{-1}$, valores que se mostram coerentes com os resultados reportados por Souto et al., na análise de vinhos tintos nacionais. ${ }^{8}$

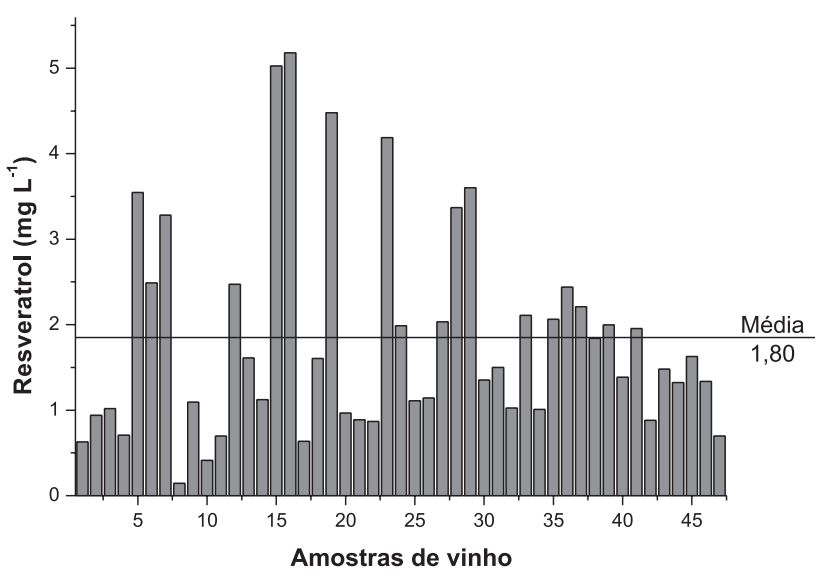

Figura 5. Representação gráfica da concentração de resveratrol nas amostras de vinho analisadas

As diferenças observadas entre vinhos de diferentes procedências podem ser devidas a um conjunto de características conhecido como terroir, que reúne aspectos relacionados com o solo e o clima, dentre outras características locais. As amostras de vinho doadas pela vinícola Miolo, por exemplo, são oriundas de três regiões distintas do Estado do Rio Grande do Sul (Campanha Gaúcha - amostra 11, Campos de Cima amostra 10 e Vale dos Vinhedos - amostra 15). Embora todas as amostras sejam da mesma varietal (Merlot), maiores quantidades de resveratrol foram observadas nas amostras procedentes do Vale dos Vinhedos. De acordo com informações climáticas do Estado do Rio Grande do Sul, ${ }^{21}$ a precipitação anual média é semelhante nestas 3 regiões. Na região do Vale dos Vinhedos, entretanto, a temperatura média é superior à das outras regiões, o que, sugerindo um maior grau de insolação, pode justificar a geração de maiores concentrações de resveratrol.

Adicionalmente, é importante considerar que, de acordo com a legislação brasileira (Lei $\mathrm{N}^{0} 7.678$ de novembro de 1988), um vinho pode ter a denominação de determinada uva se contiver, no mínimo, $75 \%$ dessa variedade. Esta exigência implica que outras variedades de uva podem ser misturas, em proporção de até $25 \%$, o que representa mais uma fonte de variação que dificulta a caracterização de vinhos, mesmo utilizando-se amostras do mesmo varietal.

Nenhuma correlação evidente foi observada entre o teor de polifenóis totais (Figura 4) e o teor de resveratrol (Figura 5). Assim, a amostra 8 , que apresenta a menor concentração de resveratrol, e a amostra 16, que apresenta o maior valor, apresentam valores intermediários de polifenóis totais.

\section{Atividade antioxidante}

Preliminarmente, a repetibilidade da metodologia foi avaliada por análise em duplicata de 8 amostras de vinho, observando-se um desvio padrão relativo médio $(3,65 \%)$ compatível com os valores relatados na literatura para este tipo de ensaio. ${ }^{9}$

Os resultados da avaliação de atividade antioxidante, expressa em mmol L-1 de Trolox por mL de vinho, são apresentados na Figura 6. Os valores mínimo e máximo deste parâmetro corresponderam a 0,83 e 2,79, respectivamente, enquanto que o valor médio foi de 2,03 mmol L ${ }^{-1}$ de Trolox por $\mathrm{mL}$ de vinho. Estes resultados são compatíveis com os resultados publicados na literatura, os quais indicam um intervalo entre 0,91 e $3,05 .{ }^{9}$

Vários estudos têm demonstrado a existência de uma boa correlação $(R=0,99)$ entre a atividade antioxidante e a concentração de polifenóis totais. ${ }^{22}$ Fazendo-se a correlação com os dados obtidos neste trabalho foi observada uma tendência geral que confirma a conexão existente entre estes parâmetros. Entretanto, existem vários casos em que a correlação é baixa, o que faz com que os coeficientes de correlação sejam muito inferiores aos relatados na literatura. Relacionando-se todas as amostras (diversas nacionalidades e espécies de uva) obtém-se um coeficiente de correlação (R) de 0,15 , enquanto que se comparando apenas uma espécie de uva, mas com diferentes safras e nacionalidades, se consegue um valor de $\mathrm{R}$ de 0,38 . No melhor dos casos, envolvendo amostras produzidas na mesma safra e com o mesmo tipo de uva, observam-se valores de $\mathrm{R}$ de 0,63 , ainda bastante baixos em relação aos antecedentes da literatura.

Para ilustrar esta falta de correlação podem-se salientar os resultados apresentados pela amostra 19, que apresenta uma capacidade 


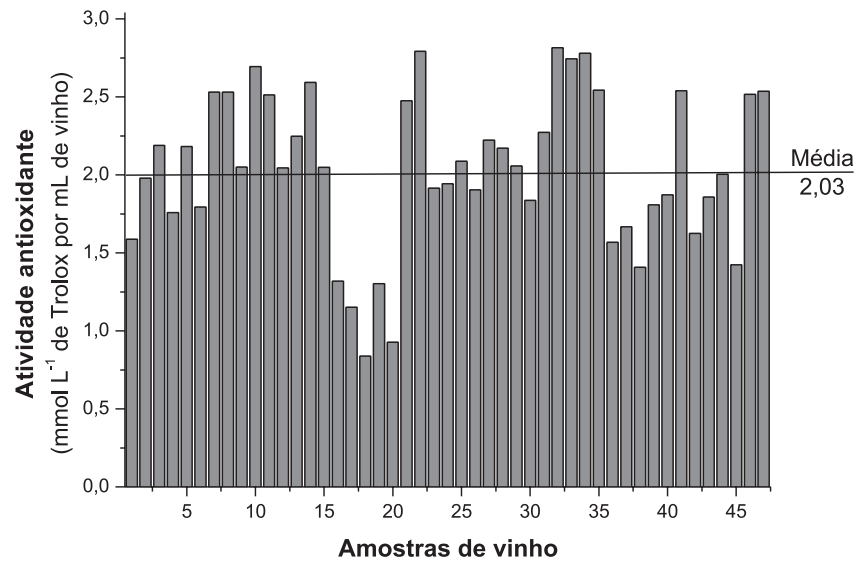

Figura 6. Representação gráfica da atividade antioxidante (equivalentes em Trolox) nas amostras de vinho analisadas

antioxidante das mais baixas e concentração de fenóis totais acima da média. Por sua vez, a amostra 34 apresenta um dos mais elevados valores de atividade antioxidante, ao mesmo tempo em que mostra um teor de espécies fenólicas bem abaixo da média.

Observações similares podem ser realizadas em relação à correlação existente entre a capacidade antioxidante e o teor de resveratrol. Neste caso, a baixa concentração relativa de resveratrol justifica a sua pouca influência na capacidade antioxidante das amostras de vinho.

De maneira geral, admite-se que grande parte da atividade antioxidante das amostras de vinho seja função das espécies polifenólicas características. Desta forma, a falta de correlação entre estes parâmetros pode ser função da falta de seletividade da técnica utilizada para a determinação de polifenóis totais (método de Folin-Ciocalteau).

Em função da correlação existente entre a capacidade antioxidane do vinho, o teor de espécies polifenólicas e o sinal espectral registrado entre 400 e $800 \mathrm{~nm}$, modelos multivariados foram desenvolvidos por regressão por mínimos quadrados parciais. Os referidos modelos foram desenvolvidos a partir da informação espectral de 42 amostras de vinho, enquanto que outras 5 foram reservadas para validação externa. Diversos sistemas de pré-processamento de sinais foram avaliados, destacando-se dados centrados na média, alisados e derivados.

O modelo de melhor desempenho foi elaborado com dados espectrais centrados na média e 4 variáveis latentes, as quais permitiram representar aproximadamente $60 \%$ da variância dos dados de atividade antioxidante, a partir de $97 \%$ da variância dos dados espectrais. Nestas condições, erros médios de previsão da ordem de $3 \%$ foram observados na fase de validação externa (Tabela 3), valores estes que se mostram compatíveis com o valor de desvio médio observado na aplicação do método de referência $(3,65 \%)$.

Tabela 3. Previsão da atividade antioxidante do conjunto de validação externa utilizando-se modelos desenvolvidos a partir de 4 variáveis latentes e espectros centrados na média $(\mathrm{CM})$ e em primeira derivada ( $1^{\mathrm{a}}$ deriv. $)$

\begin{tabular}{cccccc}
\hline Amostra & $\begin{array}{c}\text { Atividade } \\
\text { antioxidante } \\
(\mathrm{mM} \text { TEAC })\end{array}$ & $\begin{array}{c}\text { CM } \\
\left(\mathrm{mmol} \mathrm{L} \mathrm{-}^{-1}\right.\end{array}$ & $\begin{array}{c}\text { Ve Trolox previsto } \\
\text { Erro }(\%)\end{array}$ & $1^{\mathrm{a}}$ deriv. & Erro $(\%)$ \\
\hline A3 & 2,18 & 2,14 & $-1,83$ & 2,18 & 0 \\
A16 & 1,32 & 1,31 & $-0,75$ & 1,25 & $-5,30$ \\
A29 & 2,26 & 2,11 & $-6,63$ & 2,11 & $-6,63$ \\
A37 & 1,61 & 1,63 & 1,24 & 1,61 & 0 \\
A44 & 1,70 & 1,67 & $-1,76$ & 1,63 & $-4,11$ \\
Média & & & 2,44 & & 3,21 \\
\hline
\end{tabular}

$\mathrm{Na}$ Figura 3S, material suplementar, se apresenta o vetor de regressão obtido na modelagem de dados centrados na média com 4 variáveis latentes. Nessa figura, que representa as regiões espectrais de relevância para estabelecimento da modelagem, é possível observar regiões de regressão positiva (centradas em aproximadamente 425 , 495 e $590 \mathrm{~nm})$ e negativa $(535 \mathrm{~nm})$, cada uma das quais pode ser correlacionada com espécies polifenólicas de relevância, dentre as quais se destacam antocianinas, taninos e uma grande variedade de produtos de condensação entre antocianinas e flavonoides. ${ }^{16}$

Embora a capacidade de previsão do modelo se mostre adequada em relação ao conjunto de validação externa $\left(R^{2}=0,977\right)$, foi observada uma baixa correlação entre valores medidos pelo método de referência e valores previstos pelo modelo para o conjunto de calibração na fase de validação cruzada $\left(R^{2}=0,64\right.$, Figura $4 S$, material suplementar). A referida inconsistência implica que, para algumas amostras de vinho, as regiões espectrais processadas sofrem influência de espécies químicas que não contribuem com a capacidade antioxidante avaliada (interferência) ou espécies que efetivamente contribuem com a expressão de atividade antioxidante não possuem sinal espectral na região processada.

\section{CONCLUSÕES}

Praticamente todos os parâmetros físico-químicos relacionados com a atividade antioxidante de vinhos foram avaliados (cor, teor de polifenóis totais, concentração de resveratrol e atividade antioxidante), encontrando-se resultados que se mostram compatíveis com os antecedentes relatados na literatura para amostras de natureza similar.

Estudos quimiométricos fundamentados no uso de análise de componentes principais permitiram verificar certa correlação entre o grau de maturação dos vinhos e os dados espectrais registrados entre 400 e $800 \mathrm{~nm}$. Outras correlações são menos evidentes (ex. cor e maturação, cor e teor de polifenóis totais, polifenóis totais e atividade antioxidante), em razão da complexidade química das amostras de vinho e da pouca seletividade das ferramentas analíticas de caracterização (ex. método de Folin-Ciocalteau).

O uso de dados espectrais permitiu a elaboração de modelos multivariados com boa capacidade de previsão da atividade antioxidante. Modelos fundamentados no uso de dados espectrais centrados na média e elaborados a partir de 4 variáveis latentes permitiram a obtenção de erros de previsão médios da ordem de 3\%, na fase de validação externa.

\section{MATERIAL SUPLEMENTAR}

Disponível em http://quimicanova.sbq.org.br, na forma de arquivo .PDF, com acesso livre.

Na Figura 1S apresenta-se o gráfico de pesos (loadings) para as 5 primeiras componentes principais na análise de dados espectrais por rotina de PCA. Na Figura $2 \mathrm{~S}$ apresenta-se o gráfico de escores para as componentes principais 2 e 3 na análise de dados espectrais por rotina de PCA. Na Figura 3S apresentam-se os coeficientes de regressão obtidos na modelagem da atividade antioxidante com dados espectrais centrados na média e 4 variáveis latentes. Na Figura $4 \mathrm{~S}$ apresenta-se o gráfico de atividade antioxidante medida e prevista pelo modelo multivariado para o conjunto de calibração na fase de validação cruzada.

\section{REFERÊNCIAS}

1. Renaud, S.; Lorgeril, M.; Lancet 1992, 339, 1523.

2. Minussi, R. C.; Rossi, M.; Bologna, L.; Cordi, L.; Rotilio, D.; Pastore, G. M.; Duran, N ; Food Chem. 2003, 82, 409. 
3. Nepomuceno, M. F.; Mamede, M. E. O.; Macedo, D. V.; Armindo, A A.; Pereira, L. S.; Tabak, M.; Biochim. Biophys. Acta 1999, 1418, 285.

4. Halliwell, B.; Free Radical Biol. Med. 2009, 46, 531.

5. Katalinić, V.; Milos, M.; Modun, D.; Musić, I.; Boban, M.; Food Chem. 2004, 86, 593.

6. Villaño, D.; Fernández-Pachón, M. S.; Troncoso, A. M.; García-Parrilla, M. C.; Food Chem. 2006, 95, 394.

7. Gürbüz, O.; Göçmen, D.; Delen, F. D.; Gürsoy, M.; Aydin, S.; Sahin, I.; Büyükuysal, L.; Usta, M.; Food Chem. 2007, 100, 518.

8. Souto, A. A.; Carneiro, M. C.; Seferin, M.; Senna, M. J. H.; Conz, A.; Gobbi, K.; J. Food Comp. Anal. 2001, 14, 441.

9. Li, H.; Wang, X.; Li, Y.; Li, P.; Wang, H.; Food Chem. 2009, 112, 454.

10. Staško, A.; Brezová, V.; Mazúr, M.; Čertík, M.; Kaliňák, M.; Gescheidt, G.; Food Sci. Technol. 2008, 41, 2126.

11. Castillo-Sánchez, J. J.; Mejuto, J. C.; Garrido, J.; García-Falcón, S.; Food Chem. 2006, 97, 130.

12. Kuskoski, E. M.; Asuero, A. G.; Troncoso, A. M.; Mancini-Filho, J.; Fett, R.; Ciênc. Tecnol. Aliment. 2005, 25, 726.

13. Versari, A.; Boulton, R. B.; Parpinello, G. P.; Food Chem. 2008, 106, 397.
14. Castillo-Sánchez, J. X.; García-Falcón, M. S.; Garrido, J.; MartínezCarballo, E.; Martins-Dias, L. R.; Mejuto, X. C.; Food Chem. 2008, 106, 18.

15. Monagas, M.; Martín-Álvarez, P. J.; Gómez-Cordovés, C.; Bartolomé, B.; Food Sci. Technol. 2007, 40, 107.

16. Vivar-Quintana, A. M.; Santos-Buelga, C.; Rivas-Gonzalo, J. C.; Anal. Chim. Acta 2002, 458, 147.

17. Pazourek, J.; Gajdošová, D.; Spanilá, M.; Farková, M.; Novotná, K.; Havel, J.; J. Chromatogr., A 2005, 1081, 48.

18. de Oliveira, A. C.; Valentin, I. B.; Goulart, M. O. F.; Silva, C. A.; Bechara, E. J. H.; Trevisan, M. T. S.; Quim. Nova 2009, 32, 689.

19. Zhou, J.; Cui, H.; Wan, G.; Xu, H.; Pang, Y.; Duan, C.; Food Chem. 2004, 88, 613 .

20. Rodríguez-Delgado, M. A.; González, G.; Pérez-Trujillo, J. P.; GarcíaMontelongo, F. J.; Food Chem. 2002, 76, 371.

21. http://www.scp.rs.gov.br/atlas/atlas.asp? menu=26, acessada em Setembro 2009.

22. Villaño, D.; Fernández-Pachón, M. S.; Troncoso, A. M.; García-Parrilla, M. C.; Talanta 2004, 64, 501. 


\section{CARACTERIZAÇÃO ESPECTROSCÓPICA MULTIVARIADA DO POTENCIAL ANTIOXIDANTE DE VINHOS}

Wellington César Gallice, Iara Messerschmidt e Patricio Peralta-Zamora*

Departamento de Química, Universidade Federal do Paraná, CP 19081, 81531-980 Curitiba - PR, Brasil

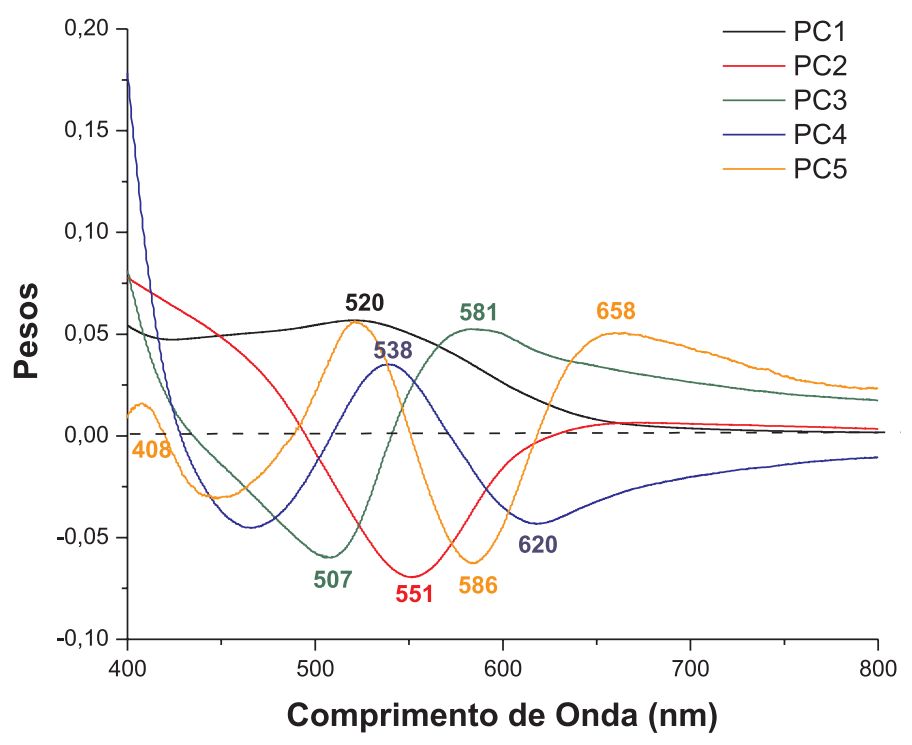

Figura 1S. Gráfico de pesos (loadings) para as cinco primeiras componentes principais na análise de dados espectrais por rotina de PCA 


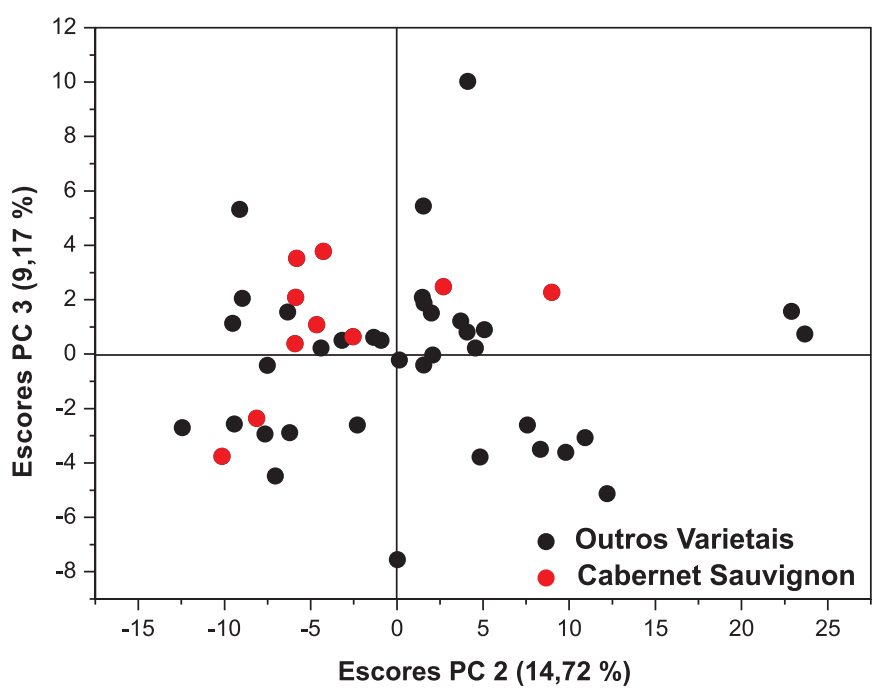

Figura 2S. Gráfico de escores para as componentes principais 2 e 3 na análise de dados espectrais por rotina de PCA

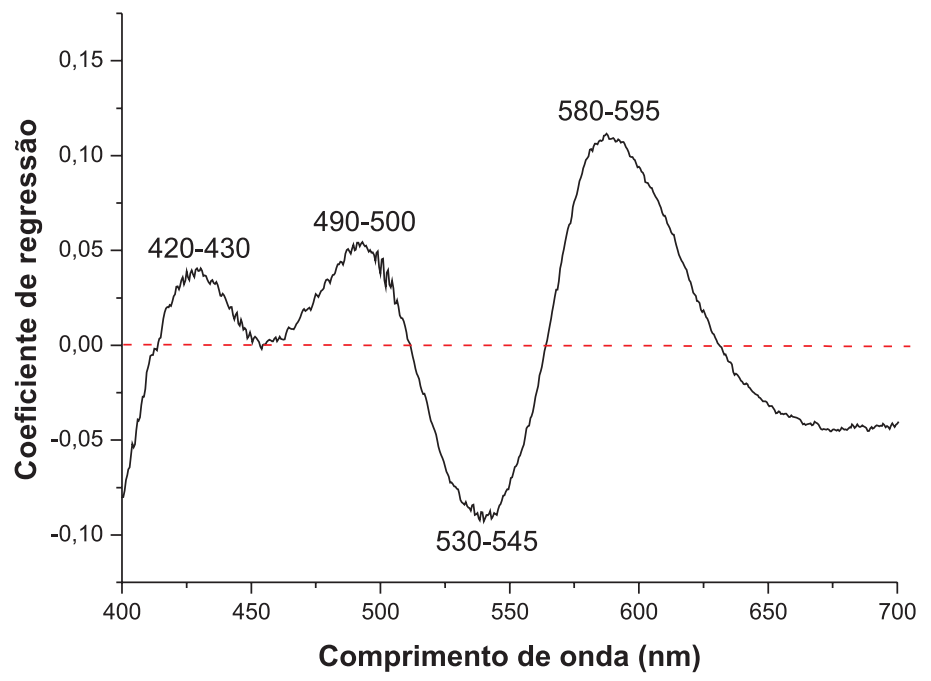

Figura 3S. Coeficientes de regressão obtidos na modelagem da atividade antioxidante com dados espectrais centrados na média e 4 variáveis latentes

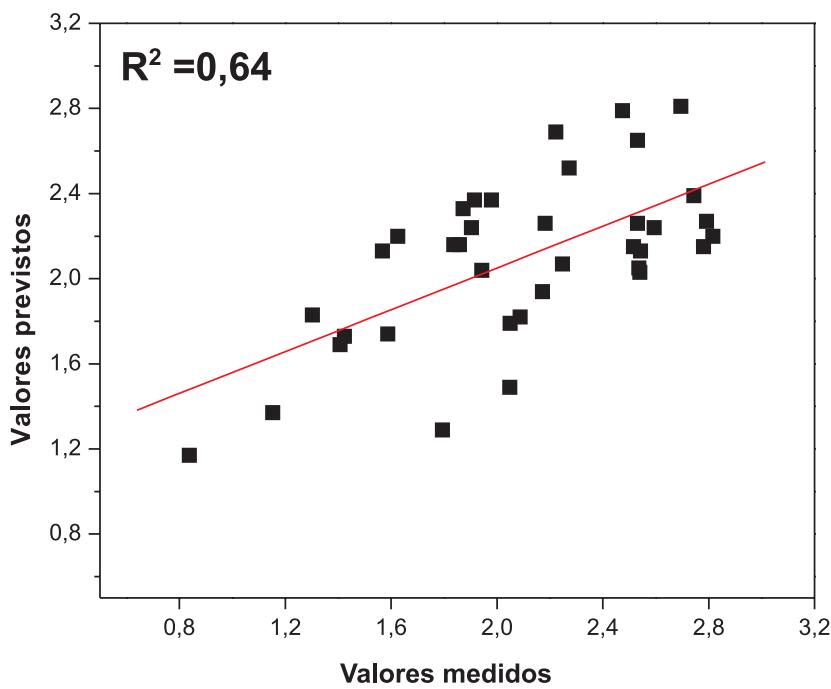

Figura 4S. Gráfico de atividade antioxidante medida e prevista pelo modelo multivariado para o conjunto de calibração na fase de validação cruzada 